\title{
Carbonaceous nanomaterials: production and application. R\&D of gas institute of NAS of Ukraine
}

\begin{abstract}
The work presents a brief review of nanotechnologies developed by the Gas Institute of NASU devoted to the production and use of carbon materials. A description is given for pilot units design for the synthesis of pyrolytic carbon, activated carbon, thermally expanded graphite, multi-wall carbon nanotubes, nanofluids based on carbon nanoparticles. All aggregates have been launched and laboratory samples produced and tested. The unique properties of carbon-based materials make it possible to apply them to a wide range of industries such as:chemical, nuclear, aerospace, automotive etc.
\end{abstract}

Keywords: carbonaceous nanomaterials, manufacturing, application
Volume 4 Issue 3 - 2018

\author{
Khovavko Al, Strativnov EV, Kozhan AP, \\ Moraru VN, Sviatenko AM, Nebesny AA, \\ Filonenko DS, Sidorenko SV \\ Gas Institute of National Academy of Science of Ukraine, \\ Ukraine
}

Correspondence: Khovavko Al, Gas Institute of Nationa Academy of Science of Ukraine, 39 Degtyarivska str., Kyiv, 03II3,Ukraine,Email ahova2005@ukr.net

Received: February 08, 2018 | Published: June 07, 2018

\section{Experimental results and their analysis}

Gas Institute of NAS of Ukraine made a series of important new researches to create technology and equipment for nanomaterials and nanofluids that can effectively be used in the newest and traditional energetics. Scientists of the Institute carried out thermodynamic study and created a pilot unit of electro thermal fluidized bed (ETFB). ETFB - a bed of electrically conductive particles in a fluidized state heated by electric current passing through its volume. It is possible to realize various processes at extremely high level of temperature (up to $2000{ }^{\circ} \mathrm{C}$ ). For the benefit of micro plasma charges in the created aggregate (one of the biggest in the Western Europe) it was implemented a continuous coating of pure quartz particles by lustrous nanopyrocarbon. ${ }^{1}$ The layer of pyrographite that capsulated quarts can be obtained in different thickness range by pyrolysis of hydrocarbons. Carbothermic reduction of silicon from quartz sand coated by pyrolytic carbon was accomplished in plasma arc discharge in the environment of vacuum with atomic hydrogen. So, we suggested to obtain pure silicon suitable for use in photovoltaic bypassing the expensive and dangerous "Siemens" chloride method (Figure 1).

Pilot, portable and autonomous installations to produce nanoflaky thermal expanded graphite (TEG) which have a great using potential

A)

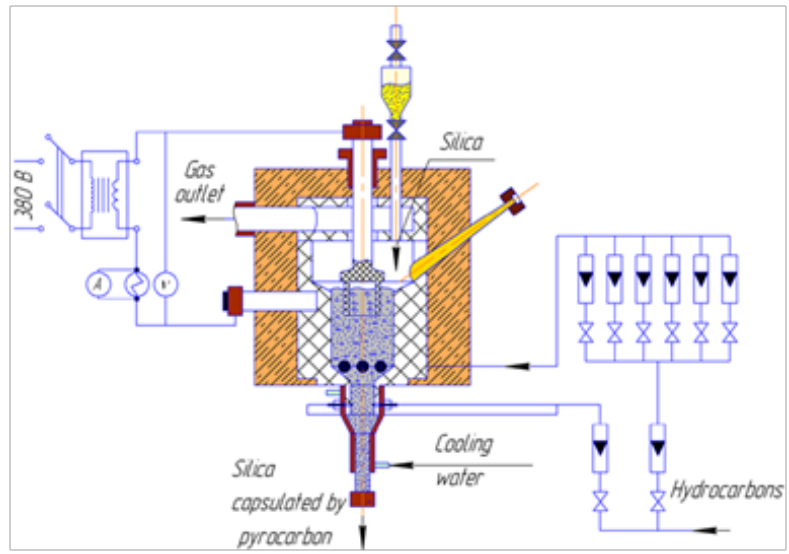

as a supersorbent for liquidation of oil and petroleum spillages have been produced. ${ }^{2}$ Methods to collect spilled oil from water and soil are elaborated, as well as methods of absorbed oil utilization and TEG-sorbent regeneration. Also, Gas Institute has designed and manufactured equipment to obtain pure TEG, which has significant prospects for use as additive to the electrodes of automotive lithiumion batteries (Figure 2) (Figure 3). In many studies thermal expanded graphite also is named as a Few Layers Graphene. Preliminary experiments on it ultrasonic treatment gave us positive results. We suggest that one sheet Graphene has been obtained. Nowadays the Gas Institute is elaborating an advanced technology, energy efficient equipment and pilot production of high quality low ash activated carbon on the base of bio raw material (shells of coconut and walnut, apricot seeds, etc) with specific surface area near $2000 \mathrm{~m}^{2} / \mathrm{g}$. Such carbon nanomaterial is very perspective for creation a newest source of electric current: lithium and zinc air batteries, super capacitors. Also enterosorbents with high organoleptic properties (flash dispersing in mouthparts) could be elaborated on the base of presented material. Such enterosorbents have a promising prognosis for the treatment of kidney diseases, diabetes, complications after the application of chemotherapy. It will be possible to create much lightweight and efficient short-cycle adsorption units for gases separating, in particular for the separation of hydrogen from gas mixtures.

B)

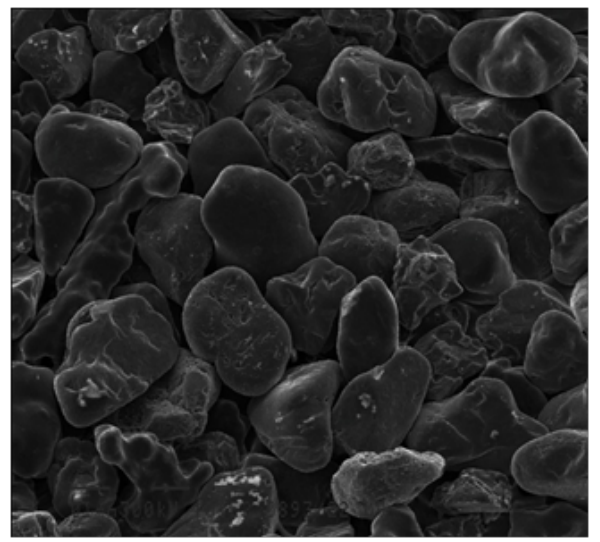

Figure I A) - reactor with ETFB for pyrocarbon synthesis; B) - quarts sand coated by pyrocarbon. 

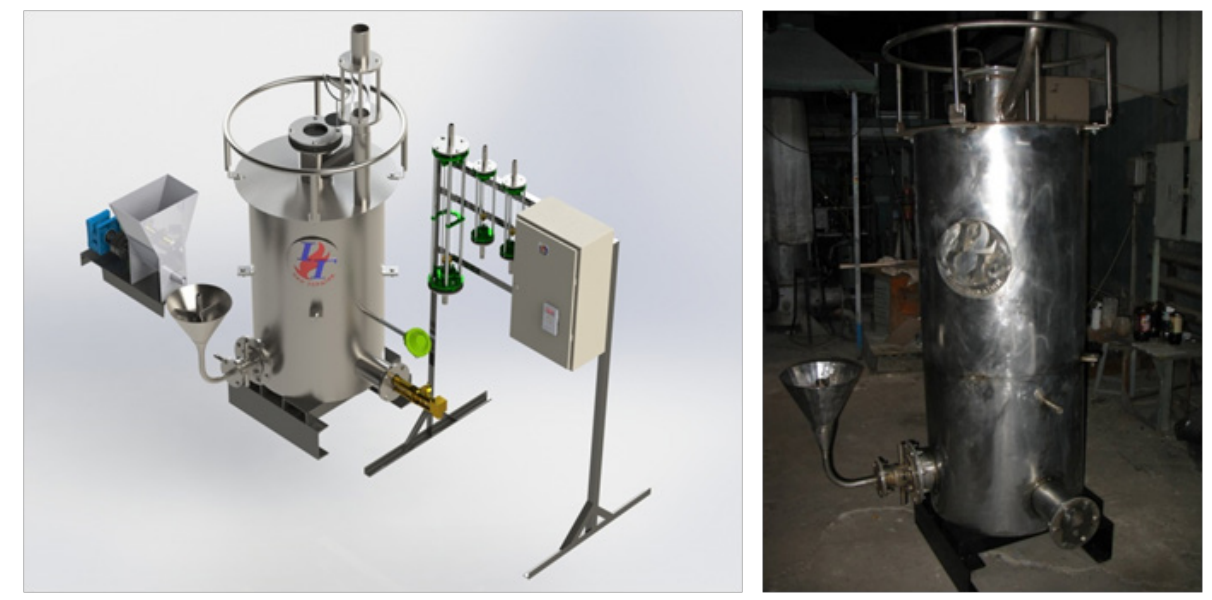

Figure 2 Semi-industrial unit for thermal expanded graphite manufacturing.
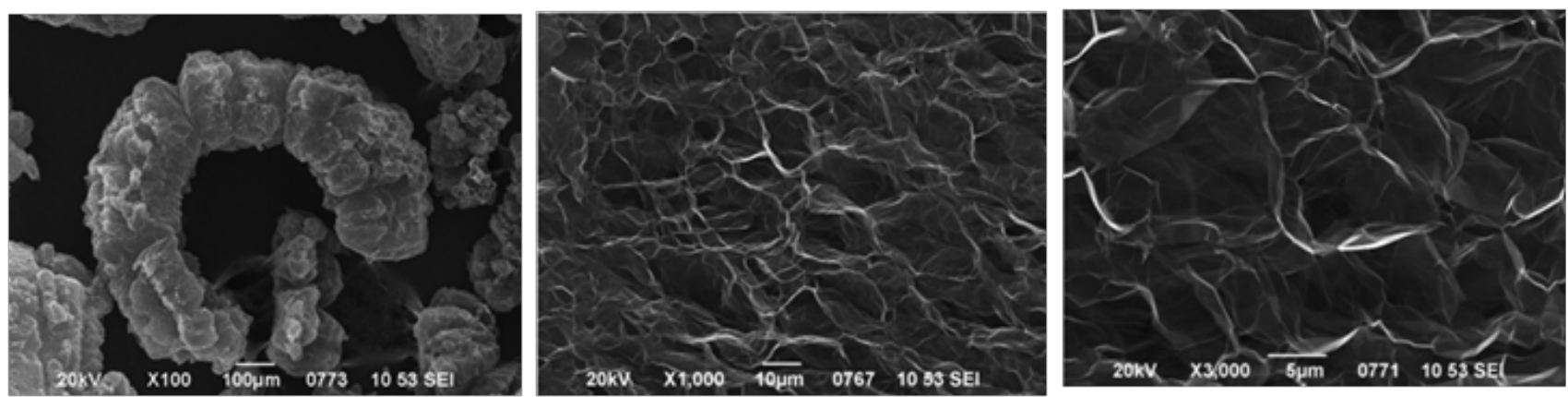

Figure 3 SEM photo of thermal expanded graphite.

On the basis of fundamental research there were obtained experimental batches of multi-wall carbon nanotubes (MWCNTs), regarded as one of the most promising materials for hydrogen storage. Multi-year experimental and theoretical studies carried out in the field of disperse metals obtaining with pre-specified carbon value are under laid at the basis of the developed technology of carbon nanotubes manufacturing. Thermodynamic modeling of phase formation while hydrocarbons catalytic decomposition is based on the software products: «GaS» and «Terra». As a result, at present, the Gas Institute has a range of the equipment allowing a deep investigation of methods to produce carbon nanotubes and catalysts for their formation (Figure 4). ${ }^{3}$ The mechanism of carbon nanomaterial formation at moderate temperatures while processing of fresh-reduced iron by products of air conversion of natural gas is considered. It is shown that under given conditions the size and the shape of the resulting carbon are depended on the temperature and the size of microscopic iron grains formed during reduction. These iron grains are the catalyzer of the reaction of carbon monoxide disproportionation. It is concluded that the formation of nucleus of the new carbon phase occurs at the contact boundaries of neighboring grains of newly reduced iron with the subsequent formation in these places of ring-shaped carbon cuffs. Nanotubes are forming as a result of further carbon crystallization and separation of iron particles from the main mass is occurring i.e., there is a fragmentation of the substance of the catalyst. According to the results of laboratory studies the optimum temperature of carbon nanotubes formation in the environment of converted gas is $600-650^{\circ} \mathrm{C}$. The evidence of the hypothesis that the mechanism of the reaction of carbon monoxide disproportionation flows through the intermediate stage of iron oxides formation is given. ${ }^{4}$

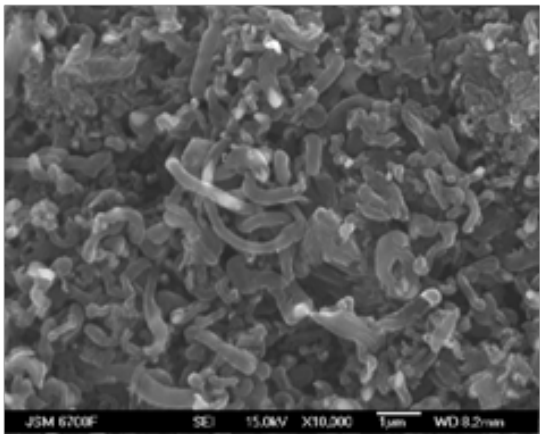

A)

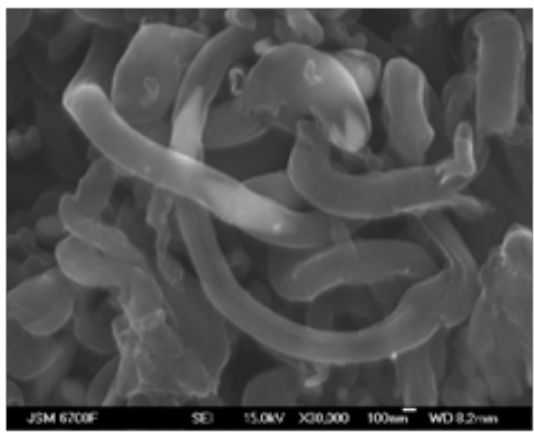

B)

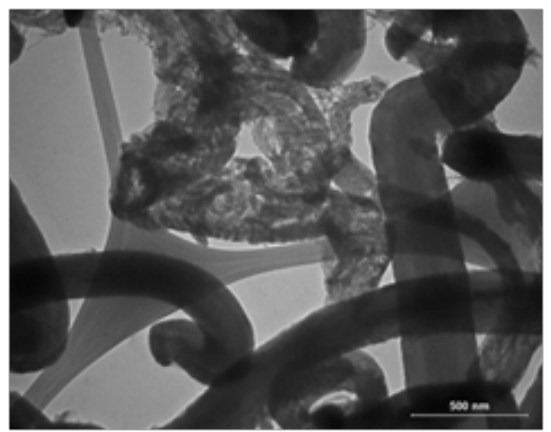

C)

Figure 4 View of MWCNTs obtained as a result of fresh-reduced iron processing by converted natural gas. A \& B) - SEM photo; C) - TEM photo. 
The purity of the resulting nanotubes reaches $95 \%$ by carbon. Calculations of the technological parameters for the manufacturing of 1 ton/day were made to realize a large-scale production of MWCNTs by developed method.The systematic study devoted to the preparation of stable nanofluids (NFs) was carried out using thermo expanded graphite, MWCNTs and Ukrainian aluminosilicates. There were developed thermal physics foundations and computerized unit for the study of critical heat flux (CHF) at nanofluids boiling. ${ }^{5}$ It was found that nanofluids have increased by $20-30 \%$ a thermal conductivity. Nanofluids using in systems with a coolant boiling can rise a CHF in 2-3,5 times (Figure 5) (Figure 6). ${ }^{6}$ It was found that NFs based on attapulgite and montmorillonite more stable to prolonged and repeated boiling-cooling and coagulation action of direct current compared with NFs -based on carbon materials, due to their different chemical nature and hydrophilic surface. A convincing proof is obtained that the CHF growth at NFs boiling in comparison with water is due to the change in the nature and microrelief of the heating surface. ${ }^{7}$ It is proved that in the presence of NFs it is possible to avoid a boiling crisis, coming suddenly, in contrast to single-phase coolants (water). Also, we performed researches devoted to the stabilization of aqueous suspensions containing MWCNTs and TEG using a wide range of stabilizers of different classes. Due to the hydrophobic nature of the MWCNTs and especially of the TEG they are difficult to stabilize. Nevertheless, our experiments allowed us to determine and select the most appropriate stabilizers of MWCNTs and TEG.

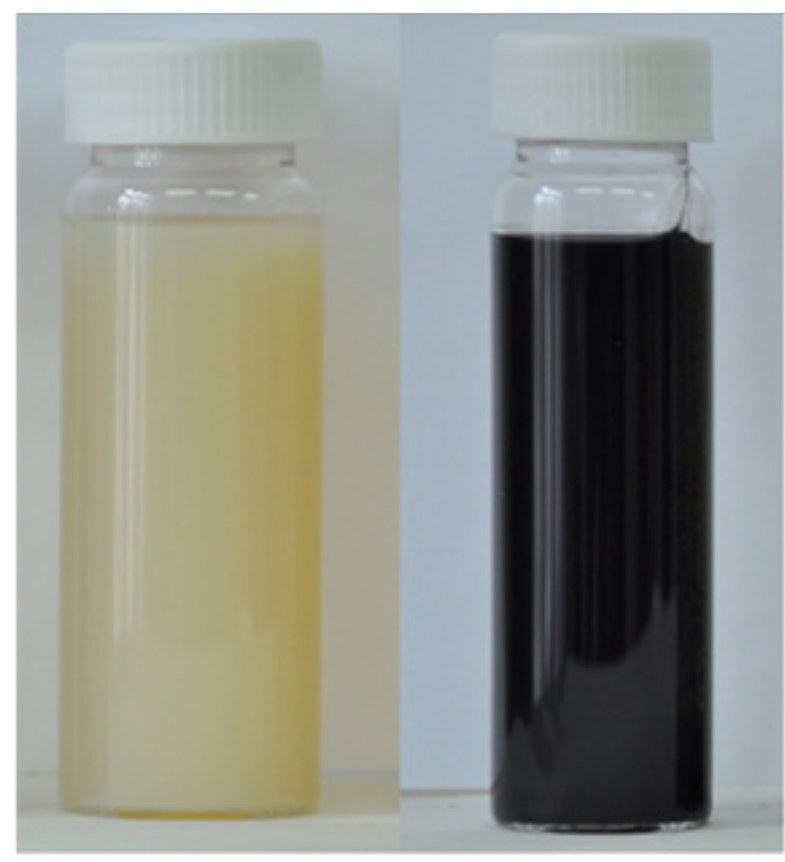

Figure 5 Nanofluids on the basis of: aluminosilicates (left); carbon nanotubes (right).
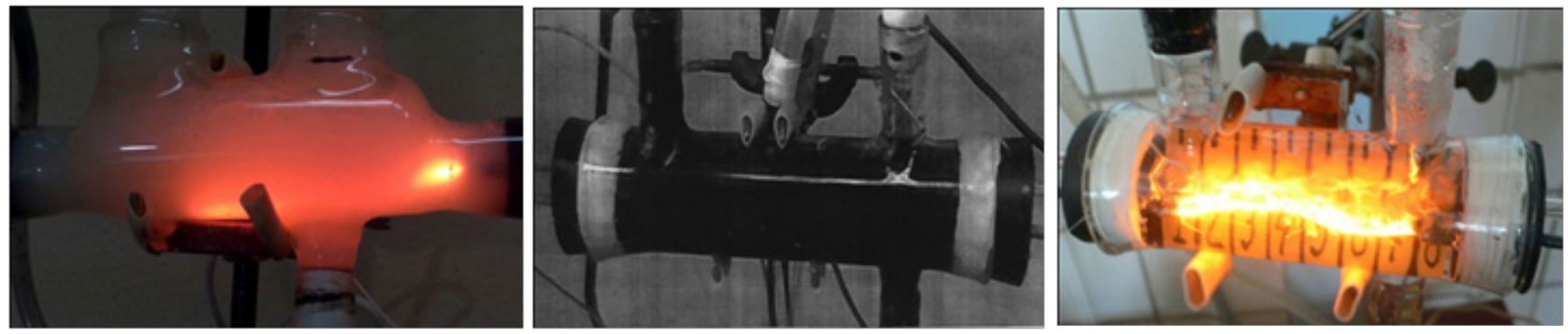

Figure 6 Experimental cell. Research of a critical heat flux at nanofluids boiling.

The resulting nanofluids are characterized by high sedimentation stability, which is measured in months and years. The concentration of nanoparticles varies within $0.1-0.5 \mathrm{vol} \%$, which is quite sufficient for a demonstration of high thermophysical properties of the coolant. The $\mathrm{pH}$ of investigated nanofluids is close to a neutral and is within the limits of 5.4 - 8.5. It has been found that the stabilization of nanoparticles by surfactants is accompanied by the hydrophilization of carbon particles, causing a sharp decrease in surface tension. The obtained results of the research allow us to make the conclusions about an increase of CHF for a number of nanofluids, the influence of initial concentrations of nanoparticles in liquids, the presence of dispersants, the nature of nanoparticles, and the coolant flow regimes on a CHF and a heat transfer coefficient. It is experimentally established that the anisotropy of particles shape influences on nanofluids thermal parameters during their boiling. Preliminary estimate shows that application of nanofluids as a heat carrier to a nuclear power plant can raise electricity production by $20 \%$ without any changes in the technological scheme.

\section{Conclusion}

The actuality of presented developments is determined by their focus on the creation of energy-efficient and resource-saving technologies. We invite all interested organizations, laboratories for collaboration with Gas Institute to test the samples of created materials for the purpose of making new strategic, competitive products.

\section{Acknowledgments}

Some results of this study were obtained under support of the National Academy of Sciences of Ukraine (Fundamental research program of NASU "Fundamental problems of nanostructured systems, nanomaterials, nanotechnology") and by State fund for fundamental research (contract F64-35).

\section{Conflict of interest}

The author declares that they have no competing interests. 


\section{References}

1. Bondarenko B, Bogomolov V, Kozhan A, et al. Development of technological foundations for pure silicon production by carbothermic reduction. International Journal of Energy for a Clean Environment. 2013;14(2-3):183-189.

2. Strativnov E. Design of Modern Reactors for Synthesis of Thermally Expanded Graphite. Nanoscale esearch Letters. 2015;10:245.

3. Bondarenko B, Sviatenko O, Kotov V, et al. Technology of carbon nanotubes in gas mixtures containing carbon monoxide. Physica Status Solidi. 2013;10(7-8):1180-1182.

4. Nebesnyi A, Kotov V, Sviatenko A, et al. Carbon nanomaterial Formation on Fresh-Reduced Iron. Nanoscale Research Letters. 2017;12:107.
5. Bondarenko BI, Moraru VN, Sidorenko SV, et al. Nanofluids for Energetics: Effect of Stabilization on the Critical Heat Flux. Technical Physics Letters. 2012;38(9):853-857.

6. Bondarenko BI, Moraru VN, Ilienko BK, et al. Study of a heat transfer mechanism and critical heat flux at nanofluids boiling International. Journal of Energy for a Clean Environment. 2013;14(2-3):151-168.

7. Bondarenko BI, Moraru VN, Sydorenko SV, et al. Nanostructured Architectures on the Heater Surface at Nanofluids Boiling and Their Role in the Intensification of Heat Transfer. Nanoscience and Nanoengineering. 2016;4(10):12-22. 